\title{
Prediction of momentum and scalar transport in turbulent swirling flows with an objective Reynolds-stress transport closure
}

\author{
B. A. Younis $\cdot$ B. Weigand $\cdot$ A. D. Vogler
}

Received: 17 February 2009/Accepted: 26 May 2009/Published online: 27 June 2009

(c) The Author(s) 2009. This article is published with open access at Springerlink.com

\begin{abstract}
The accurate prediction of turbulent swirling flows requires the use of a differential Reynolds-stress transport model to close the time-averaged Navier-Stokes equations. The performance of such model is largely determined by the way in which the fluctuating pressurestrain correlations are approximated. A number of alternative approximations are available, all of which depend explicitly on the mean vorticity tensor. Such dependence renders a constitutive relation inconsistent with the principle of Material Frame Indifference (MFI). In this paper, an objective model (i.e. one which is consistent with MFI) for the pressure-strain correlations is presented. This model, which was developed using Tensor Representation Theory, has fewer terms than the conventional alternatives and is therefore easier to implement in computational codes. Moreover, the model was calibrated to correctly reproduce the relative stress levels in both free and wall-bounded flows without the need to employ wall-damping corrections. The performance of this model is assessed using experimental data from both weakly- and strongly-swirled jets. Comparisons are also made with results obtained using three widely-used alternative models for the pressure-strain correlations. It is found that the objective model, although simpler in formulation than the others, yields results that are generally in closer correspondence with the data. The paper also reports on the prediction of mass transfer in a swirling jet. The case considered was that of a co-axial, strongly-
\end{abstract}

\section{B. A. Younis $(\square)$}

Department of Civil \& Environmental Engineering,

University of California, Davis, CA 95616, USA

e-mail: bayounis@ucdavis.edu

B. Weigand · A. D. Vogler

Institut für Thermodynamik der Luft- und Raumfahrt (ITLR),

Universität Stuttgart, 70569 Stuttgart, Germany swirled flow with an outer annular air stream and an inner helium jet. Swirl was imparted to the outer stream only. The concentration of helium was predicted using a differential scalar-flux transport closure. Close agreement was obtained with the measured concentrations. Analysis of the predicted mass fluxes revealed that the turbulent diffusivity is strongly anisotropic in this flow.

Keywords Turbulence modeling - Swirl - Scalar fluxes . Pressure-strain correlations · Grid Convergence Index

\section{Introduction}

In many flows of practical interest, swirl is imparted to a turbulent jet in order to enhance the level of turbulence activity and thereby increase the rate of transfer of momentum, concentration and temperature between the jet and its surroundings. In gas turbine combustors, for example, the increased mixing rates improve the efficiency of the combustion process and reduce harmful emissions. If the degree of the imparted swirl is sufficiently strong, then the adverse pressure gradients that arise may be sufficient to cause local flow reversal [1]. This has the added advantage of forming regions of slow-moving flow which serves to stabilize the flame.

In a number of recent studies, Large-Eddy Simulations [2] and Direct Numerical Simulations [3, 4] were used to study the patterns of flow and mixing which occur in recirculating swirling flows. Such simulation strategies, while having the potential of becoming practical simulations tools with the rapid advances in computing power, remain somewhat impractical for routine engineering design which still relies on the solution of Reynoldsaveraged equations with associated turbulence closures. In 
this context, the prediction of the effects of swirl on turbulent transport of mass and momentum has proved beyond the capabilities of simple Eddy-viscosity closures and it is now generally accepted that the accurate prediction of these effects requires the use of a complete Reynolds-stress transport closure. If mass transport is present, a model for the turbulent mass fluxes which is more physically based than the simple gradient-transport (Fick's law) model would be required to accurately capture the effects of swirl on scalar transport. The combination of models for the Reynolds stresses and the turbulent scalar fluxes most likely to be successful in a wide range of complex swirling flow would therefore appear to be one in which differential transport equations are solved for each of the six non-zero components of the Reynolds-stress tensor and for all three components of the mass fluxes. The equations contain a number of unknown correlations which require modeling in terms of known or knowable parameters. For the case of the Reynolds stresses, amongst these unknown correlations, and perhaps the one that is most difficult to model, is the long-time correlation between the fluctuating pressure field at a point and the fluctuating mean rate of strain at the same point. These are often referred to as the pressurestrain correlations and their importance in the prediction of swirling flows has long been recognized. Launder and Morse [5] were the first to attempt to predict the development of a swirling jet with a complete Reynolds-stress transport model. Their computations, however, produced results that were the exact opposite of the experimental observations. Specifically, the spreading rate of the swirling jet, which is a sensitive indicator of the intensity of turbulent mixing, was predicted to be smaller than that of the equivalent, non-swirling jet. Gibson and Younis [6] traced the problem to the model used for the pressurestrain correlations and proposed an alternative weighting for the various components that constitute this model. This yielded satisfactory results for confined and unconfined swirling jets. However, the model of [6], in common with all other models for the pressure-strain correlations used in the prediction of swirling flows, depended explicitly on the mean vorticity tensor. This quantity is not 'objective' in the sense that its value depends on the observer's frame of reference. Consequently, models which have the vorticity enter in their formulation are not consistent with the principle of Material Frame Indifference (MFI). In effect, such models may yield different results depending on whether the computations were performed in inertial or non-inertial frames. Many in the turbulence modeling community consider this to be an undesirable feature in a model (e.g. [7]) but, so far, no proposals for a practical alternative have been put forward and evaluated in flows involving swirl. In this study, we present a recently-proposed model for the pressure-strain correlations which is consistent with the principle of MFI and which has yielded good results in the benchmark case of a flow in a channel rotated about its spanwise axis [8]. This model is extended here to weaklyand strongly-swirled flows and is validated by comparisons with experimental data. The relative performance of this model is assesses by comparisons with three alternative pressure-strain models which do not satisfy the requirements of MFI. Because swirling flows often involve mixing between streams of different densities, the model evaluation was conducted using data from a co-axial jet consisting of an inner helium jet and an outer air stream. Swirl was imparted to the outer stream only and the density variation was not sufficiently large for buoyancy effects to be important. The turbulent mass fluxes were computed using a differential scalar-flux transport model which was also extended here to swirling flows. The details of the models used are presented in the next section.

\section{Mathematical formulation}

The simulations were obtained by solving the threedimensional Navier-Stokes equations which, for constant property steady flows, are written as:

$\frac{\partial U_{i}}{\partial x_{i}}=0$,

$U_{j} \frac{\partial U_{i}}{\partial x_{j}}=\frac{\partial}{\partial x_{j}}\left(v \frac{\partial U_{i}}{\partial x_{j}}-\overline{u_{i} u_{j}}\right)-\frac{1 \partial p}{\rho \partial x_{i}}$,

$U_{j} \frac{\partial C}{\partial x_{j}}=\frac{\partial}{\partial x_{j}}\left(\frac{v \partial C}{S c \partial x_{j}}-\overline{u_{i} c}\right)$

where $U_{i}$ is the mean velocity, $C$ is the helium mass fraction, $\overline{u_{i} u_{j}}$ is the Reynolds-stress tensor and $\overline{u_{i} c}$ is the massflux tensor. Repeated indices imply summation. Both the weakly- and strongly-swirled jets considered here were axisymmetric and were thus more conveniently analyzed using the cylindrical-polar coordinates system. Thus, all three velocity components $(U, V$ and $W)$ are finite but the gradients of all the dependent variables in the circumferential direction were zero.

The Reynolds stresses were obtained from the solution of differential transport equations in which they are the dependent variables. These equations are of the general form:

$\frac{D \overline{u_{i} u_{j}}}{D t}=D_{i j}+P_{i j}+\Phi_{i j}-\epsilon_{i j}$

In Eq. $4, D / D t$ is the total derivative which represents the rate of transport of $\overline{u_{i} u_{j}}$ by the mean flow (advection), $D_{i j}$ is the rate of transport by combined molecular, turbulent and fluctuating pressure processes (diffusion), $P_{i j}$ is the rate of production of $\overline{u_{i} u_{j}}, \Phi_{i j}$ is the fluctuating pressure-strain correlations and $\epsilon_{i j}$ is the rate of dissipation by viscous action. 
The terms $C_{i j}$ and $P_{i j}$ are exact and in no need of approximation. Transformation of the $C_{i j}$ terms to cylindrical-polar coordinates yields a number of non-gradient terms which are treated here in a manner consistent with the requirement of invariance under rotation of coordinates [6]. The turbulent diffusion processes $D_{i j}$ are collectively modeled using the gradient-transport hypothesis which makes the rate of diffusion of a component of $\overline{u_{i} u_{j}}$ proportional to the gradient of the stress component itself:

$D_{i j}=\frac{\partial}{\partial x_{k}}\left(C_{s} \overline{-} \bar{\epsilon} \frac{\partial}{\epsilon u_{l}} \frac{\partial \overline{u_{i} u_{j}}}{\partial x_{k}}\right)$

where $k$ is the turbulence kinetic energy and $\epsilon$ is its rate of dissipation. Transformation of the model of Eq. 5 from the Cartesian-tensor form into the cylindrical-polar coordinate systems also produces a number of non-gradient terms. These are implemented as 'sources' in the discretized equations and their contributions turn out to be crucial in ensuring the equality of the normal-stress components $\overline{v^{2}}$ and $\overline{w^{2}}$ on the jet's axis. Another term requiring approximation is the viscous dissipation term. With the assumption that the turbulence Reynolds number is high (as would be the case in swirling flows away from the wall), the viscous dissipation is considered to be isotropic and hence $\epsilon_{i j}=2 / 3 \delta_{i j} \epsilon$. The scalar dissipation was obtained from the solution of the equation:

$U_{j} \frac{\partial \epsilon}{\partial x_{j}}=\frac{\partial}{\partial x_{k}}\left(C_{\epsilon}{ }_{\epsilon}^{-} \overline{u_{k} u_{l}} \frac{\partial \epsilon}{\partial x_{l}}\right)+\frac{\epsilon}{k}\left(C_{\epsilon 1} P_{k}-C_{\epsilon 2} \epsilon\right)$

where $P_{k}\left(=-\overline{u_{i} u_{j}} \partial U_{i} / \partial x_{j}\right)$ is the rate of production of turbulence kinetic energy. The coefficients in Eq. 6 were assigned their usual values viz. $C_{\epsilon}=0.18, C_{\epsilon 1}=1.45$ and $C_{\epsilon 2}=1.90$. The fluctuating pressure-strain correlations $\left(\phi_{i j}\right)$ have the form:

$\Phi_{i j}=\overline{\frac{p^{\prime}}{\rho}\left(\frac{\partial u_{i}}{\partial x_{j}}+\frac{\partial u_{j}}{\partial x_{i}}\right)}$

As was already mentioned in the Sect. 1, a number of alternative models for $\Phi_{i j}$ exit and all have in common the assumption that $\Phi_{i j}$ depends the turbulence anisotropy $b_{i j}$, its invariants (usually only the second invariant $I I_{b}$ ), the mean rate of strain tensor $S_{i j}$ and the mean vorticity tensor $W_{i j}$, thus:

$\Phi_{i j}=f\left(b_{i j}, b_{i j} b_{i j}, S_{i j}, W_{i j}\right)$

where

$$
\begin{aligned}
S_{i j} & =\frac{1}{2}\left(\frac{\partial U_{i}}{\partial x_{j}}+\frac{\partial U_{j}}{\partial x_{i}}\right) \\
W_{i j} & =\frac{1}{2}\left(\frac{\partial U_{i}}{\partial x_{j}}-\frac{\partial U_{j}}{\partial x_{i}}\right) \\
b_{i j} & =\frac{\overline{u_{i} u_{j}}}{\overline{u_{q} u_{q}}}-\frac{1}{3} \delta_{i j} \\
I_{b} & =b_{i j} b_{i j}
\end{aligned}
$$

The representation theorems for isotropic symmetric tensor-valued functions of symmetric tensors by [9] provide the necessary tensor generators and their coefficients for the representation of $\Phi_{i j}$ according to the dependencies of Eq. 8 [10]. The result is:

$$
\begin{aligned}
\Phi_{i j}= & -\left(C_{1} \epsilon+C_{1}^{*} P_{k}\right) b_{i j}+C_{2} \epsilon\left(b_{i k} b_{k j}-\frac{1}{3} b_{k l} b_{k l} \delta_{i j}\right) \\
& +\left(C_{3}-C_{3}^{*} I I_{b}^{\frac{1}{2}}\right) k S_{i j} \\
& +C_{4} k\left(b_{i k} S_{j k}+b_{j k} S_{i k}-\frac{2}{3} b_{k l} S_{k l} \delta_{i j}\right) \\
& +C_{5} k\left(b_{i k} W_{j k}+b_{j k} W_{i k}\right)
\end{aligned}
$$

As already mentioned, the presence of the vorticity in Eq. 8 renders the model for $\Phi_{i j}$ inconsistent with the principle of Material Frame Indifference (MFI) since the vorticity itself is not independent on the frame of reference used to define it. The most direct way of conforming the model to the principle of MFI is therefore by excluding $W_{i j}$ from Eq. 8 and then applying the representation theorem of [9] with the now smaller list of dependencies. The outcome of this process is an expression similar to that of Eq. 10 except that the coefficient $C_{5}$, which provides the weighting for the vorticity-dependent contribution, would be zero. This was done in [11]. The final result is a simpler model with one less coefficient to determine. Moreover, by setting the coefficient $C_{3}$ to 0.8 , the model reduces to an expression which satisfies exactly the result from Rapid Distortion Theory for homogeneous turbulence subjected to sudden distortion. The remaining coefficients of the objective model are determined, as usual, by matching the relative stress levels implied by the model equations to values obtained by experiments on homogeneous and non-homogeneous flows with and without shear. This was done in [8] and the resulting values that are appropriate to the objective-model coefficients are listed in Table 1. The model based on these coefficients will hereafter be referred to as 'DY'. Table 1 also gives the values of the coefficients appropriate to the three most widely used models, namely the two models of Launder et al. [12] (LRR1 and the truncated version LRR2 which is also referred to as the Isotropization-of-Production model) and that of Speziale et al. [10].

When applied in confined flows, both of the LRR models require corrections to compensate for the excessive

Table 1 Coefficients of pressure-strain models

\begin{tabular}{llllllll}
\hline Model & $C_{1}$ & $C_{1}^{*}$ & $C_{2}$ & $C_{3}$ & $C_{3}^{*}$ & $C_{4}$ & $C_{5}$ \\
\hline DY & 4.0 & 2.0 & 0 & 0.8 & 2.0 & 0.6 & 0 \\
LRR1 & 3.0 & 0 & 0 & 0.8 & 0 & 1.75 & 1.31 \\
LRR2 & 3.6 & 0 & 0 & 0.8 & 0 & 1.20 & 1.20 \\
SSG & 3.4 & 1.8 & 4.2 & 0.8 & 1.3 & 1.25 & 0.4 \\
\hline
\end{tabular}


weighting given to data from homogeneous flow in local equilibrium in determination of the models' coefficients. This correction involves the use of a 'wall-damping' function whose specification can become problematic in complex geometries. In the present computations, the corrections used in conjunction with the LRR models are those proposed by [13]. In contrast, neither the SSG nor the DY models requires the use of such corrections as their coefficients were determined with reference to more recent data on relative stress levels in wall-bounded flows and from flows which are removed from local equilibrium.

The turbulent mass fluxes were also obtained from the solution of differential transport equations in which they are the dependent variables. These equations are of the general form:

$\frac{D \overline{u_{i} c}}{D t}=\frac{\partial}{\partial x_{k}}\left(C_{c}-\overline{-} \bar{\epsilon} \overline{u_{k} u_{l}} \frac{\partial \overline{u_{i} c}}{\partial x_{k}}\right)+P_{i c, 1}+P_{i c, 2}+\pi_{i c}$

where the diffusion coefficient $C_{s}$ is taken equal to 0.15 [14]. The terms on the right-hand side of Eq. 11 represent, respectively: turbulent diffusion modeled along the lines of Daly and Harlow's gradient-transport hypothesis, production of $\overline{u_{i} c}$ due to mean-concentration gradients, production due to mean-velocity gradients and the fluctuating pressure-concentration-gradients correlations. As is usual for high turbulence Reynolds number flows, the viscous dissipation term was neglected.

The production terms require no approximation and are given by:

$P_{i c, 1}=-\overline{u_{i} u_{k}} \frac{\partial C}{\partial x_{k}}$

$P_{i c, 2}=-\overline{u_{k} c} \frac{\partial U_{i}}{\partial x_{k}}$

The fluctuating pressure-concentration-gradients correlations were modeled as the sum of two terms one of which accounts for the purely turbulent interactions and another

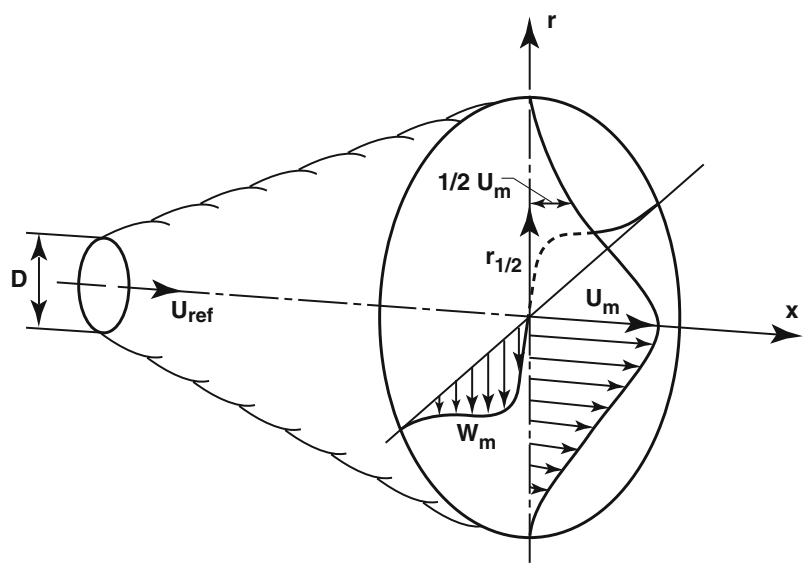

Fig. 1 Definitions and coordinates of free swirling jet which accounts for the interactions between the mean strain and the fluctuating quantities:

$\pi_{i c}=\pi_{i c, 1}+\pi_{i c, 2}$

where

$\pi_{i c, 1}=-C_{1 c} \frac{\epsilon}{k} \overline{u_{i} c}$

$\pi_{i c, 2}=-C_{2 c} P_{i c, 2}$

Following [14], $C_{1 c}$ is taken as 2.85 and $C_{2 c}$ as 0.55 .

The governing equations, transformed into cylindricalpolar coordinates, were solved using a finite-volume method. The SIMPLE algorithm was used to couple the solution of the continuity and momentum equations. The computational method utilizes a staggered grid to ensure, on one hand, that the iterative solution of the three momentum equations and the pressure did not produce checker-board oscillations and, also, to ensure that no instabilities developed due to uncoupling between the momentum equations and the relevant shear-stress components. Spatial discretization of the advection and diffusion terms was achieved with the second-order accurate
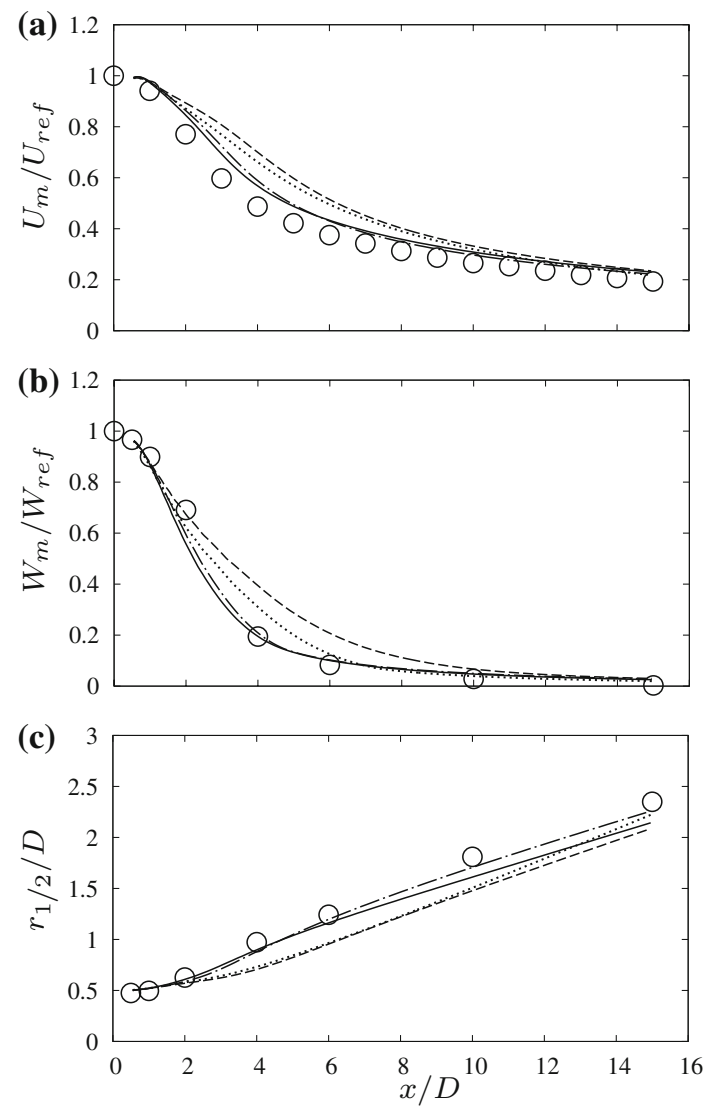

Fig. 2 Predicted and measured streamwise variation of a centerline axial velocity, b maximum swirl velocity, $\mathbf{c}$ jet half-width. 00000 : experimental data; —_-: DY; .......: LRR1; - - - - -: LRR2; -......: SSG 

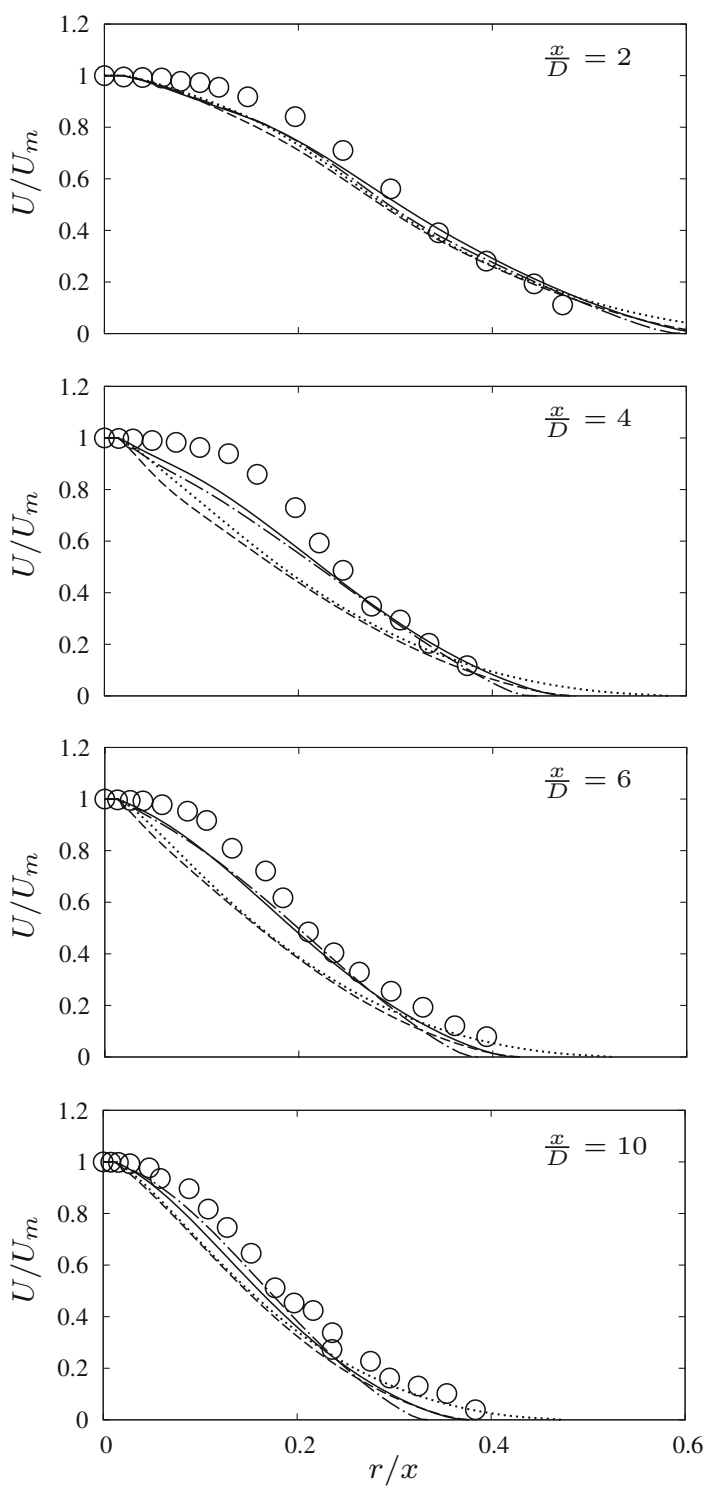

Fig. 3 Predicted and measured profiles of mean axial velocity. Symbols are as in Fig. 2

QUICK scheme [15] applied to all the dependent variables. The iterative solution of the equations proceeded from assumed initial conditions until the normalized sum of the residuals of all variables fell to below $10^{-3}$.

The numerical accuracy of the predictions were assessed in two different ways: by overlaying the predictions obtained with successively finer grids and noting the differences between them and, more quantitatively, by using the Grid-Convergence Index (GCI) method. The method is explained in detail in [16] and the steps involved in its implementation are listed in the Appendix. The method involves the determination of an index and an associated error bar which quantifies the numerical uncertainty that is present in the solutions obtained with a particular grid (typically the finest grid). The method involves comparisons
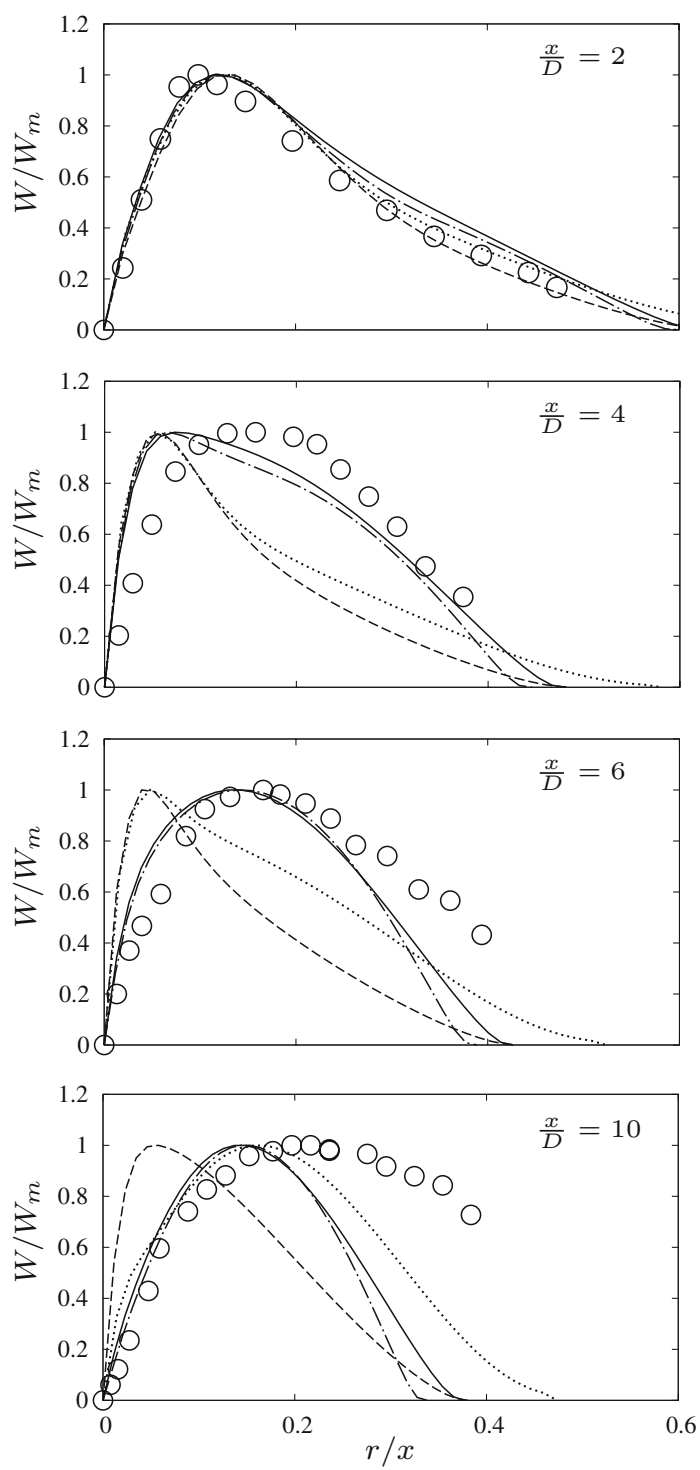

Fig. 4 Predicted and measured profiles of mean swirl velocity. Symbols are as in Fig. 2

between the solutions obtained with three different grids, and then by using the theory of generalized Richardson extrapolation. The details can be found in the original reference and the results obtained with this method for the case of the strongly-swirled jet are presented in the next section.

\section{Results and discussion}

\subsection{Weakly-swirled free jet}

The flow considered is that of an axisymmetric jet discharged into stagnant surroundings (Fig. 1). Swirl is imparted at the origin and the swirl number $S$ (defined as the ratio of the tangential to the axial momentum fluxes) 

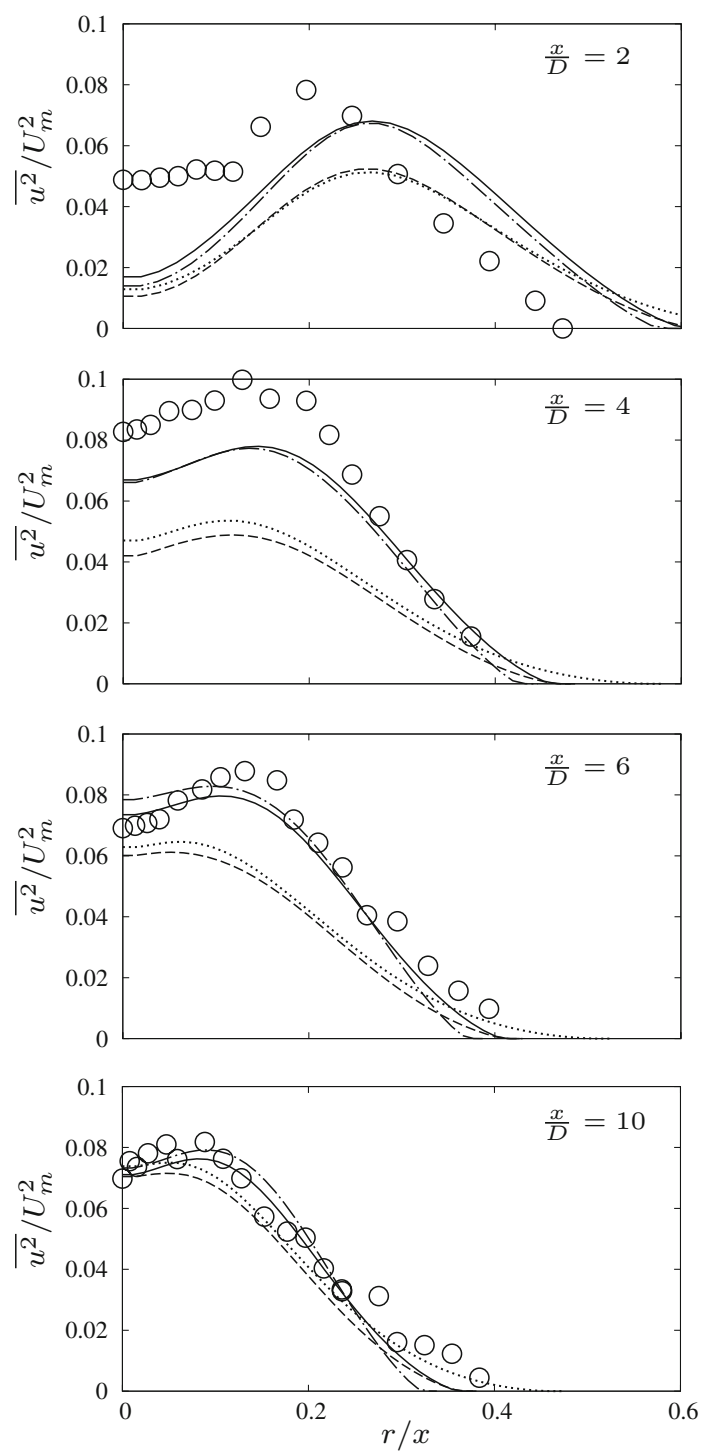

Fig. 5 Predicted and measured normal-stress component $\overline{u^{2}}$. Symbols are as in Fig. 2

was equal to 0.4 . This is sufficiently small for the swirling flow to remain unidirectional with no flow reversal. The computations were started at distance $x / D=0.5$ from measured profiles of mean velocities and turbulent stresses. A total of 34 nodes were used in the cross-stream direction. The grid was allowed to expand in the direction of flow so as to exactly match the physical width of the jet. The streamwise grid spacing was limited to $2 \%$ of the local shear-layer width. Iterations were performed at each forward step to ensure that all governing equations were simultaneously satisfied.

Figure 2 shows the development of bulk flow parameters with streamwise distance. The maximum values of both the streamwise velocity $(U)$ and the swirl velocity $(W)$ decay very rapidly under the effect of increased
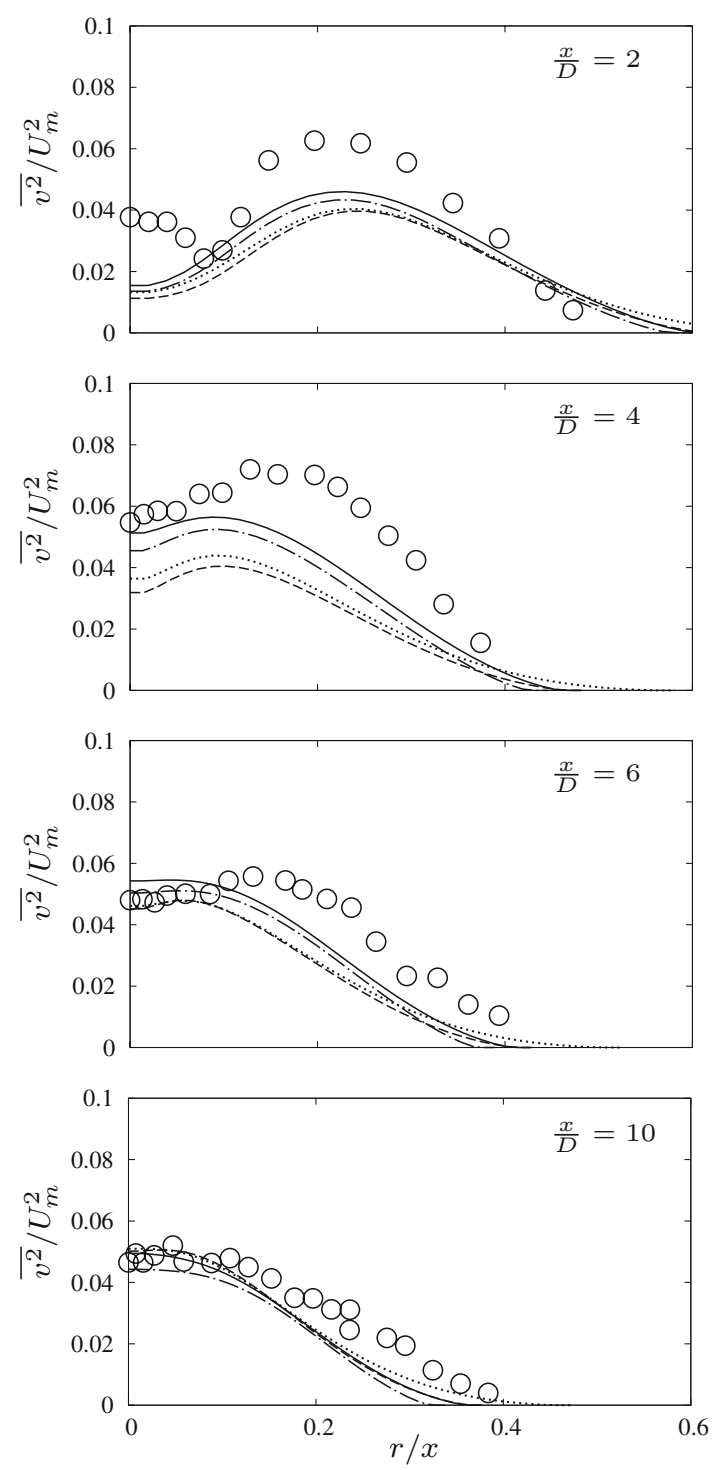

Fig. 6 Predicted and measured normal-stress component $\overline{v^{2}}$. Symbols are as in Fig. 2

turbulence mixing due to swirl while the jet's half width (defined as the distance from the centerline to the location where $\left.U=0.5 U_{\max }\right)$ increases as the jet expands by vigorous entrainment of the surrounding fluid. The DY and the SSG models come closest to predicting the measured behavior. Note, in particular, the early stages of the jet's development where the LRR models fail to capture the rapid decrease in maximum velocities in response to the adverse axial pressure gradient. The DY and the SSG models also comes closest to predicting the cross-stream distribution of the axial and swirl velocities, as can be seen in Figs. 3 and 4. The predicted and measured normal stresses are compared in Figs. 5, 6, 7. Here, too, it is evident that both the DY and SSG models obtain better predictions of the normal stresses across the entire shear 

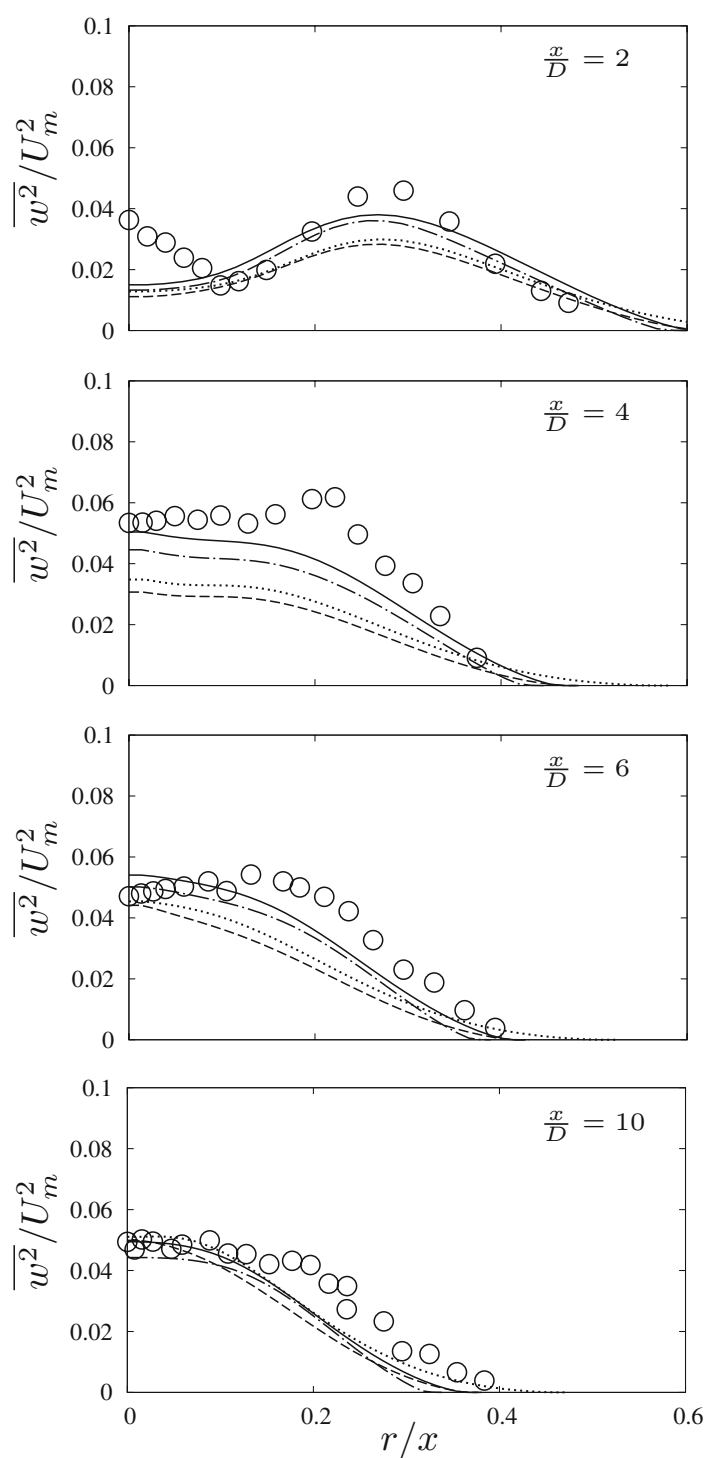

Fig. 7 Predicted and measured normal-stress component $\overline{w^{2}}$. Symbols are as in Fig. 2

layers. This is most notable in the near-field zone where the effects of swirl are most intense. Further downstream, at $x / D=10$, all model yield approximately similar predictions as the jet evolves closer to the non-swirling asymptotic state. The predicted and measured profiles of $\overline{u w}$ are compared in Fig. 8. This component of shear stress does not enter into the momentum equations in this axisymmetric, boundary-layer-like flow. Its importance, however, is that it appears in the expressions for $\Phi_{i j}$ and hence errors in its prediction are reflected in computed levels of the shear stress components $\overline{u v}$ and $\overline{v w}$. Indeed, the incorrect results obtained by Launder and Morse [5] were directly due to their predicted values of $\overline{u w}$ being predominantly negative while the measurements showed
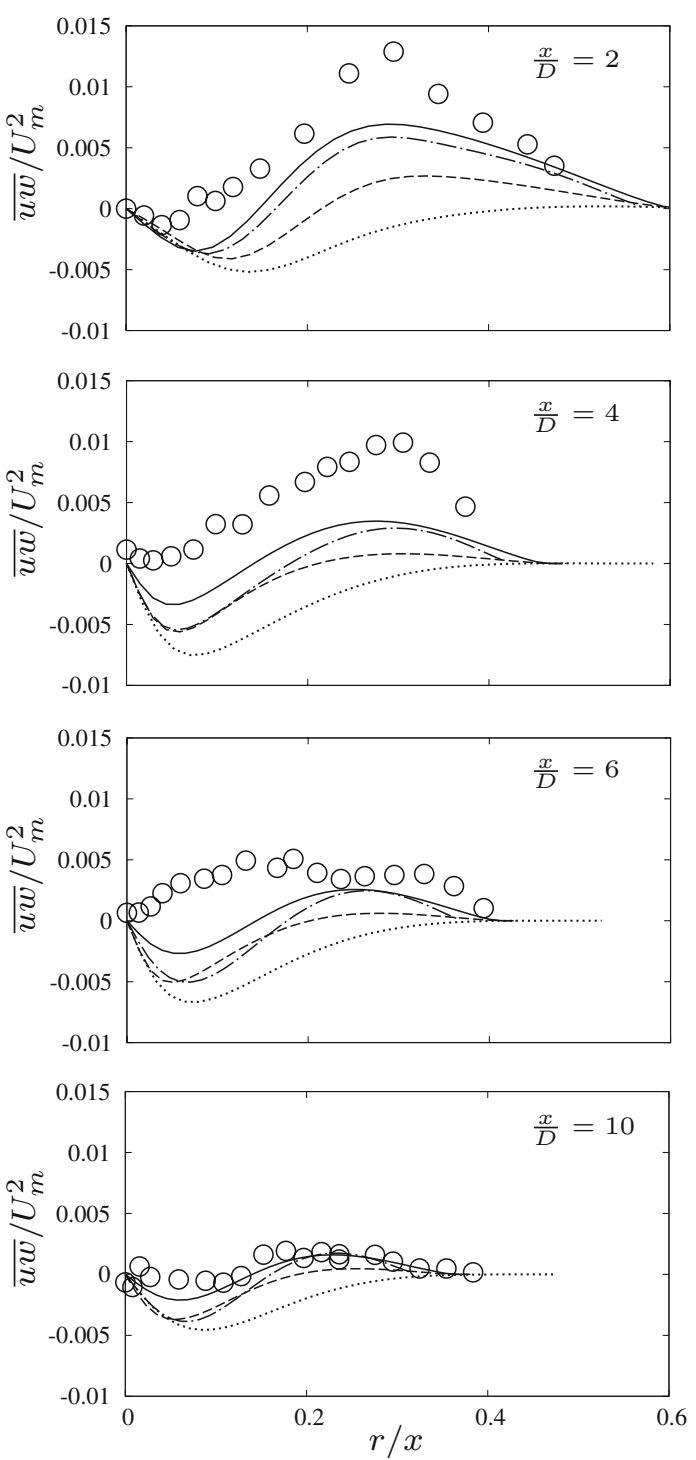

Fig. 8 Predicted and measured shear-stress component $\overline{u w}$. Symbols are as in Fig. 2

these to be positive. Figure 8 shows the extent of this problem in both the LRR models. It also shows that the DY model results comes closest to matching the measured behavior especially close to the jet's axis where the errors in the other models' results are most significant. The predicted and measured profiles of $\overline{u v}$ and $\overline{v w}$ are compared with the measurements in Figs. 9 and 10, respectively. It is again clear that the DY and SSG model yield distinctly better results than the LRR models. The shearstress component $\overline{u v}$ enters the momentum equations via the radial diffusion term for U-momentum. The higher values of this component predicted with the SSG and DY models are consistent with the faster spreading rates and decay of maximum axial velocity observed Fig. 2. 

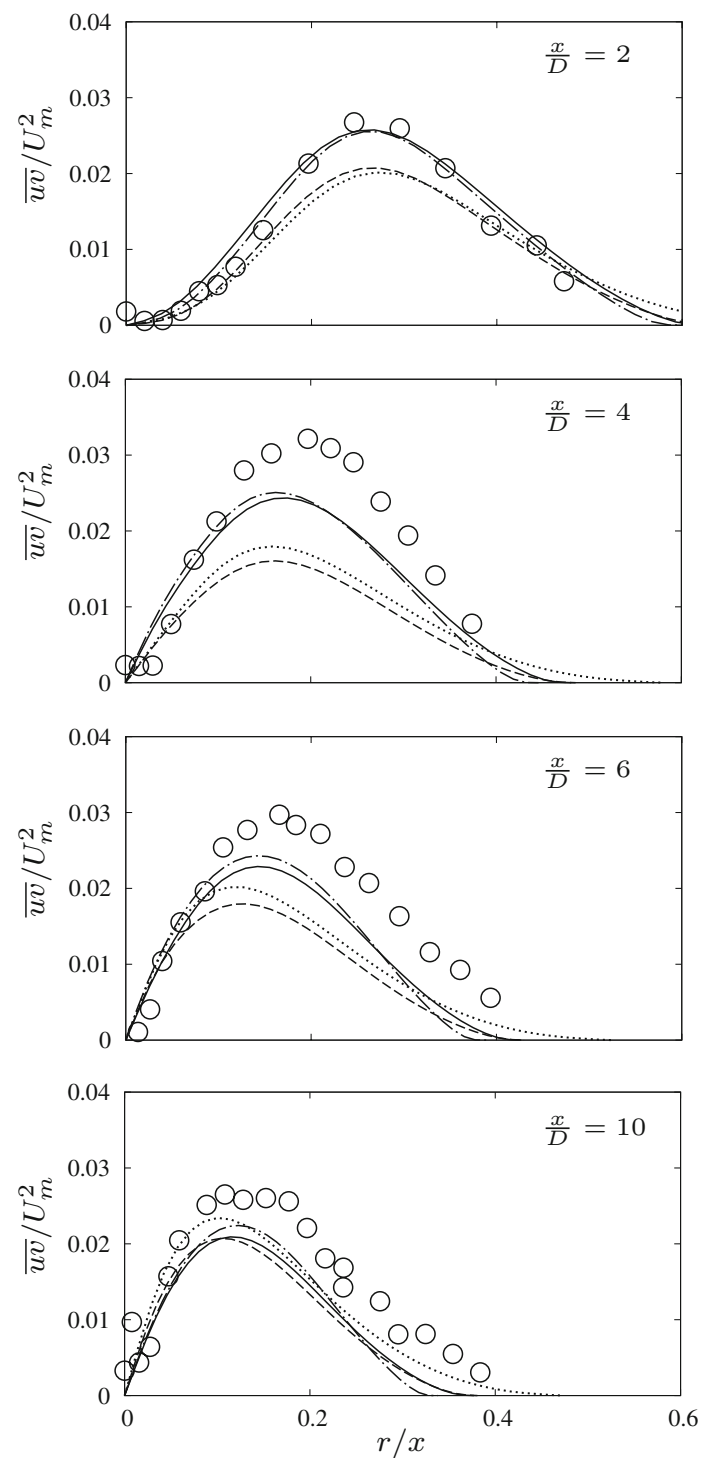

Fig. 9 Predicted and measured shear-stress component $\overline{u v}$. Symbols are as in Fig. 2

\subsection{Strongly-swirled confined jet}

The data used for the strongly-swirled test case are from the confined-flow experiments of So et al. [17]. Air was introduced into a circular chamber of radius $R=62.5 \mathrm{~mm}$ through a co-axial nozzle at inlet. Swirl was introduced to the outer stream via guide vanes (Fig. 11). The inner stream was non-swirling and this effectively prevented flow reversal along the centerline even though the swirl number was high $(S=2.25)$. Comparisons are also made with data from the experiments of Ahmed and So [18] in which helium formed the inner non-swirling jet while the outer swirling stream was air. Measurements of mean concentration were reported and these will be used to verify the present differential transport model for the turbulent scalar fluxes.
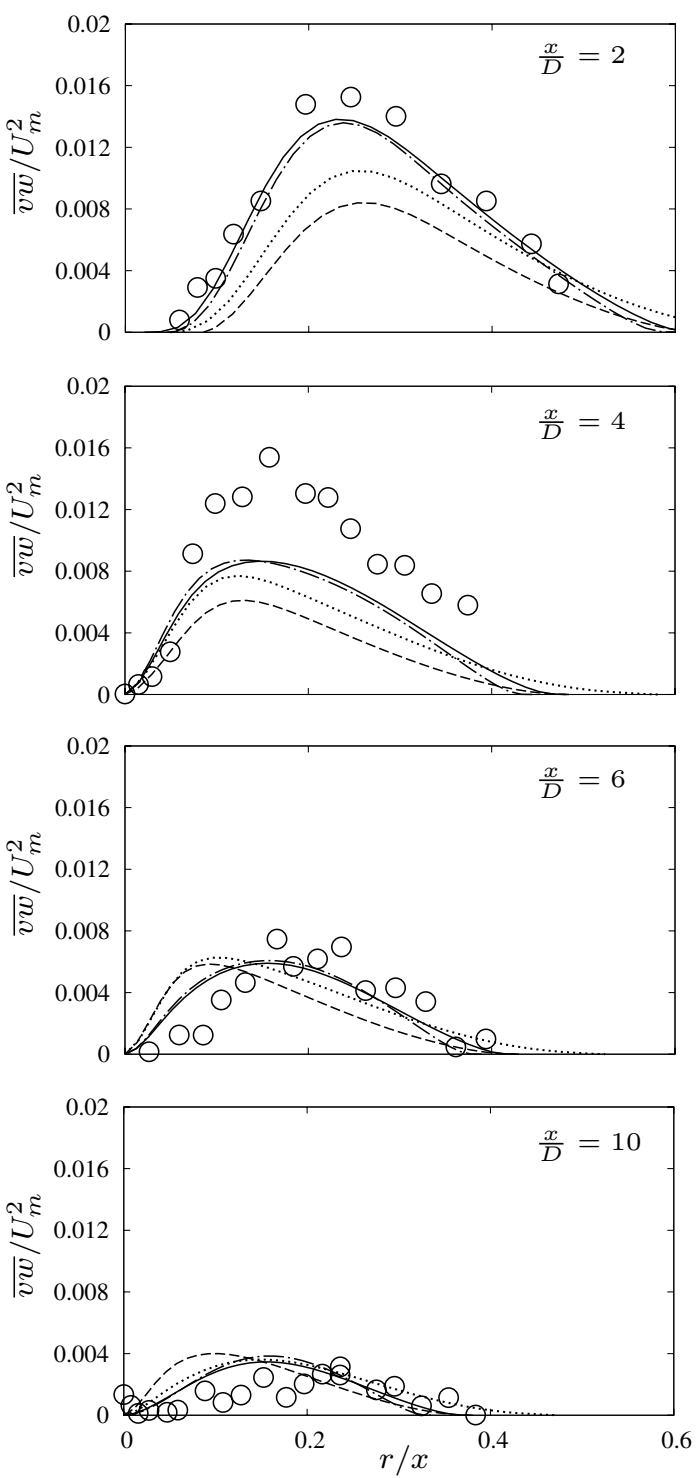

Fig. 10 Predicted and measured shear-stress component $\overline{v w}$. Symbols are as in Fig. 2
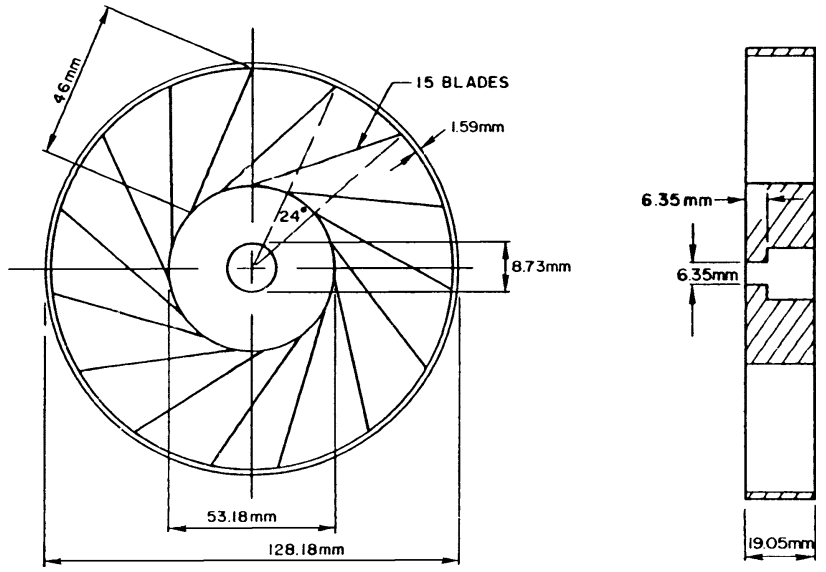

Fig. 11 Details of inlet nozzle showing center jet and guide vanes 


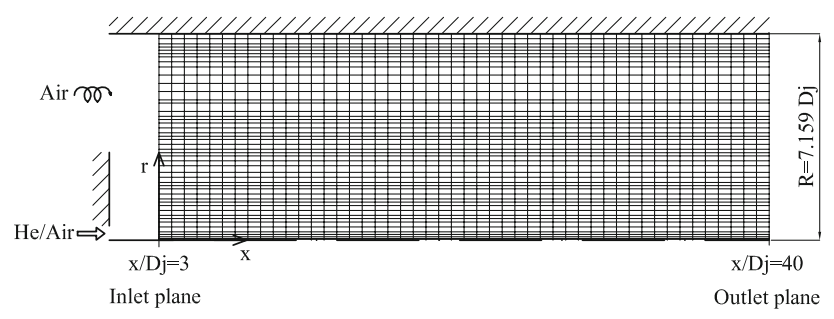

Fig. 12 Computational domain
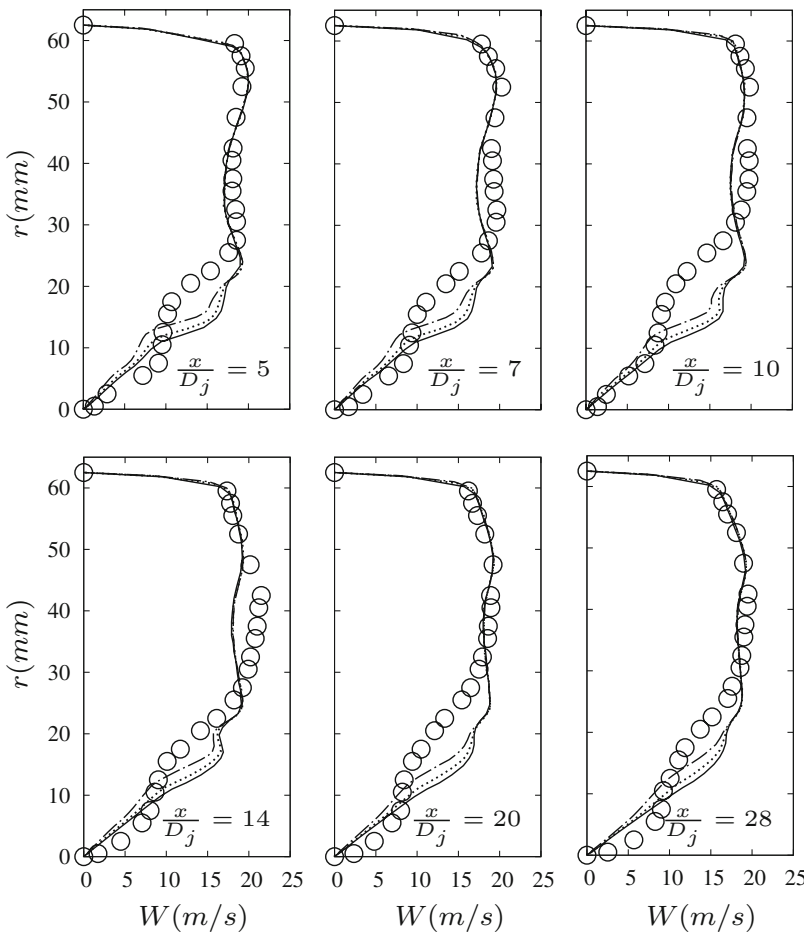

Fig. 13 Effects of grid density on mean velocity component $W$. ०००००: experimental data; ——: $50 \times 50$ grid; $\cdots \cdots \cdot 62 \times 62$ grid; -.....-: $74 \times 74$ grid

The inlet conditions were specified at $x / D_{j}=3.0$. This is the first location downstream from the inlet where experimental data were available (Fig. 12).

To check numerical discretization errors, computations were performed on three different grids having $50 \times 50$, $62 \times 62$ and $74 \times 74$ nodes in the streamwise and radial directions. The results for the radial distribution of swirl velocity are shown in Fig. 13. It is very clear that the truncation errors that remain in the $74 \times 74$ grid are quite small throughout the length of the computation domain with the maximum values occurring close to the exit. A more quantitative indication of grid dependence in the results is shown in Fig. 14. Plotted there is the predicted variation of centerline axial velocity with distance as obtained with the $74 \times 74$ grid. Superimposed on the results are error bars which represent the numerical errors as estimated with the Grid Convergence Index method. The
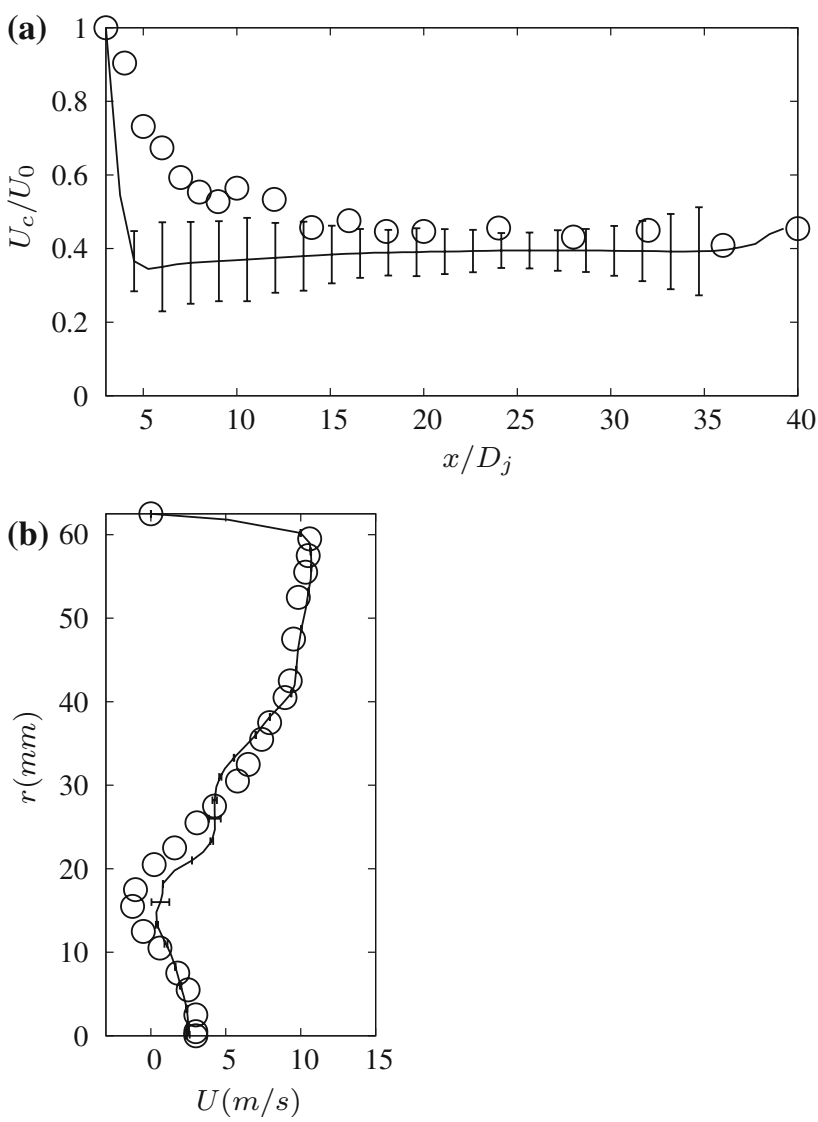

Fig. 14 a Decay of centerline velocity and $\mathbf{b}$ axial velocity profile at $x / D_{j}=14$ with error bars obtained from the GCI method.

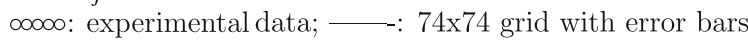

errors are clearly within acceptable bounds and do not present a plausible explanation for the observed wide departure between predictions and measurements. It is worth noting in this context that a closer agreement with the measurements is obtained by using a very coarse grid $(24 \times 24)$ but the numerical errors present were quite substantial. The predicted and measured axial velocity profiles are compared in Fig. 15. The results, which are presented for all the axial stations for which measurements have been reported, show a remarkably unchanging profile characterized by essentially constant centerline velocity, a momentum deficit (a wake) at a radial location which corresponds to the radius of the co-axial nozzle and a uniform outer flow. All models for $\Phi_{i j}$ manage to reproduce the main features of the axial flow though they appear to underestimate the extent of the wake. The swirl velocity profiles are compared in Fig. 16. The swirl, which was initially introduced only to the outer flow, is seen to diffuse toward the jet's axis where the flow appears to be in solid-body rotation. The predicted and measured profiles of axial and tangential turbulence intensities are compared in Figs. 17 and 18. The LRR models overestimate the axial intensity near the outer walls of the confining chamber despite the use 

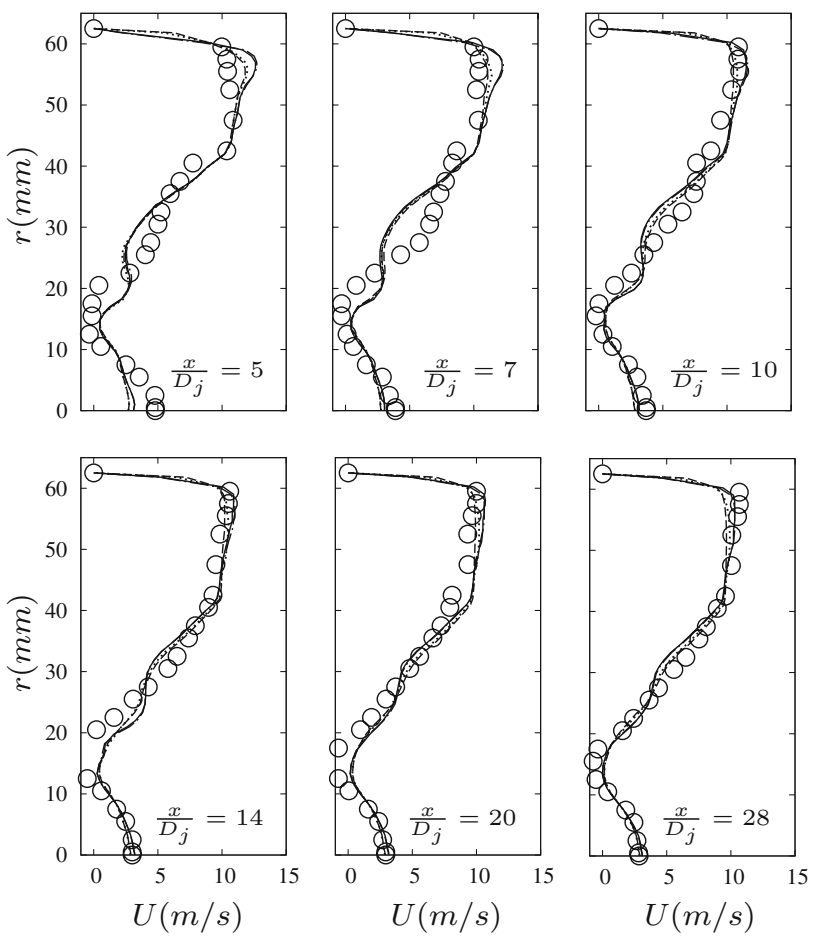

Fig. 15 Predicted and measured cross-stream profiles of the mean flow velocity component $U$ obtained with different pressure-strain models. Symbols are as in Fig. 2

of a wall-reflections model. In contrast, both the SSG and DY models obtain better predictions near the wall and throughout the rest of the flow. In contrast, none of the models tested succeeds in predicting the measured values of $\overline{u w}$, as can be seen from Fig. 19. Except close to the wall, where the models yield values that are of the same order as the measurements, the predicted values are two orders of magnitude lower than the measurements. No satisfactory explanation can be found for such large discrepancy. The stress component $\overline{u w}$ enters into the axial diffusion of the swirl velocity and the $\mathrm{W}$-profiles in Fig. 16 do not suggest a mis-match between measurements and predictions of the extent shown. It should also be noted that the measured values of $\overline{u w}$ appear to reach a maximum on the centerline when in fact they should be tending to zero there.

Predicted and measured cross-stream profiles of mean concentration are compared in Fig. 20. In the present simulations, the helium mass fraction is treated as a passive scalar and therefore variable-density effects are not taken into consideration. Ahmed and So [18] show from analysis of their measurements that the buoyancy effects associated with the helium jet are negligible and do not in any way influence the subsequent flow development downstream of the exit nozzle. The most striking feature of the distribution in Fig. 20 is the rapid trend in helium concentration to zero outside of the core region. Ahmed and So [18] comment on
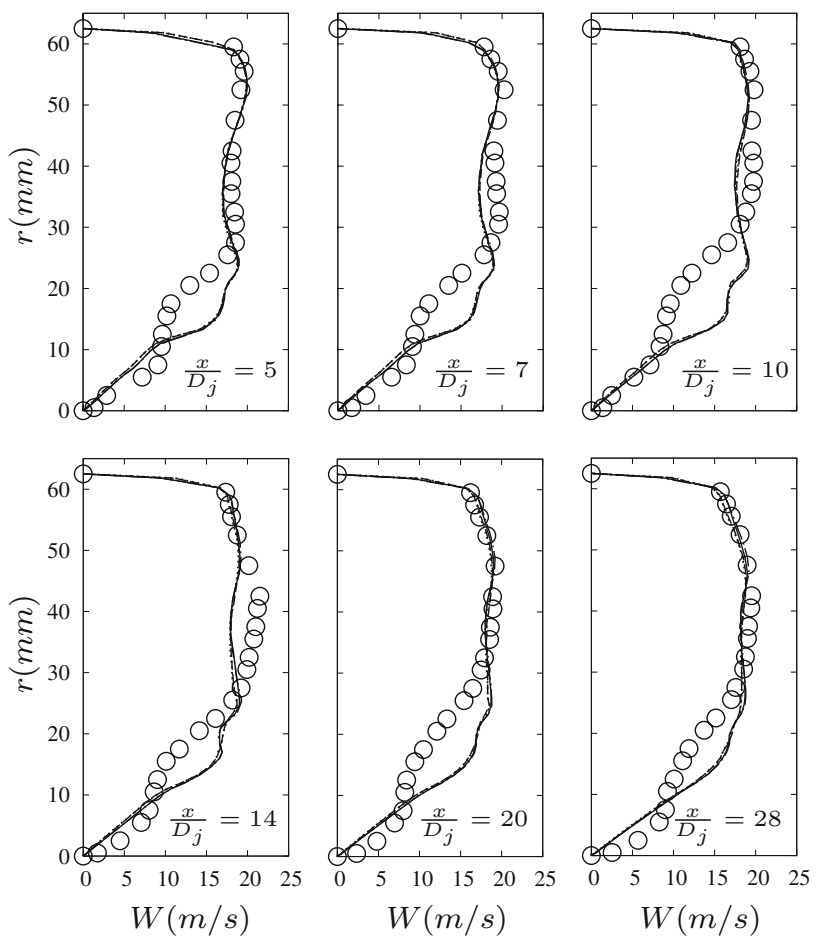

Fig. 16 Predicted and measured cross-stream profiles of the mean flow velocity component $W$ obtained with different pressure-strain models. Symbols are as in Fig. 2

the absence of migration of helium particles from the inner non-swirling jet, toward the outer stream. This is also apparent in the predictions of the mass-flux components $\overline{u c}$ and $\overline{v c}$, shown in Figs. 21 and 22. No measurements of these quantities are available for comparison. While predictions with the simple gradient-transport model (Fick's law) for the turbulent scalar fluxes were not obtained, it is nevertheless instructive to analyze the present results to determine the implications of the use of such a model. A key assumption in Fick's law is that the turbulent diffusivity is an isotropic quantity which is proportional to the eddy viscosity via a constant Schmidt number $\left(S c_{t}\right)$. Since the turbulent mass fluxes were obtained here from the solution of their own differential transport equations, it is possible to test this assumption by examining the ratio:

$\frac{S c_{x}}{S c_{r}}=\frac{\overline{u c} /(\partial C / \partial x)}{\overline{v c} /(\partial C / \partial r)}$

The results are shown in Fig. 23. Departures of the ratio of $\sigma_{x} / \sigma_{r}$ from unity signify anisotropy effects. These effects are clearly very significant throughout the development length and across the entire radial extent of the flow. Recently developed algebraic scalar-flux models (e.g. [19, 20]) are capable of obtaining anisotropic turbulent diffusivities and thus have the potential of obtaining predictions that are comparable to those of the scalar-transport closure. 

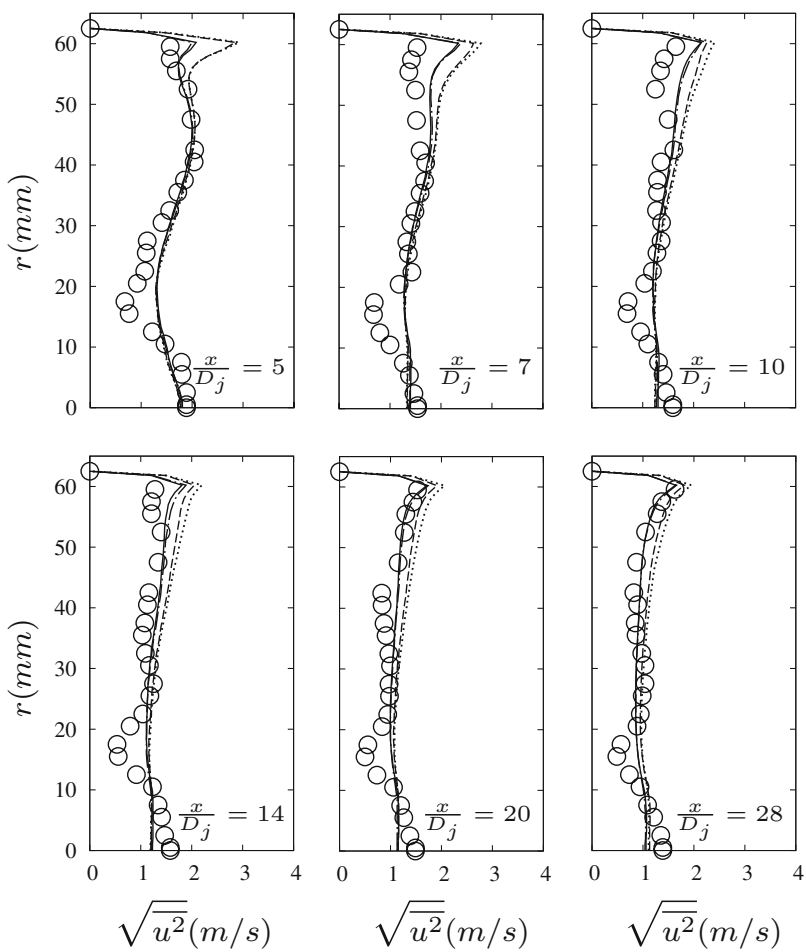

Fig. 17 Predicted and measured cross-stream profiles of streamwise turbulence intensity $\sqrt{\overline{u^{2}}}$ obtained with different pressure-strain models. Symbols are as in Fig. 2
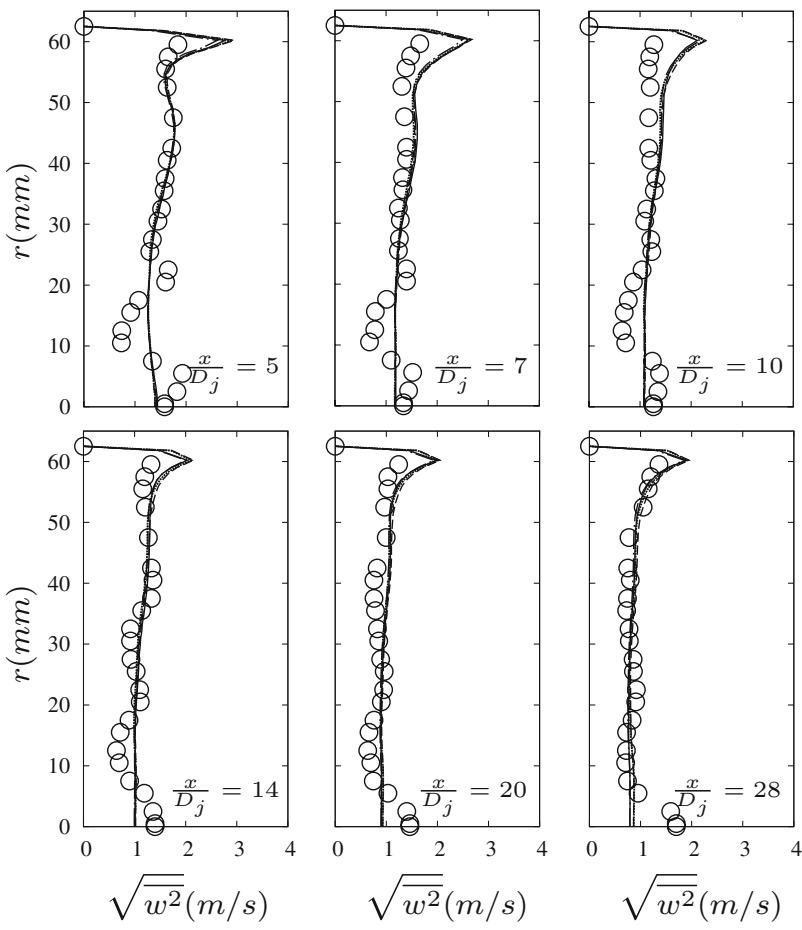

Fig. 18 Predicted and measured cross-stream profiles of circumferential turbulence intensity $\sqrt{\overline{w^{2}}}$ obtained with different pressurestrain models. Symbols are as in Fig. 2
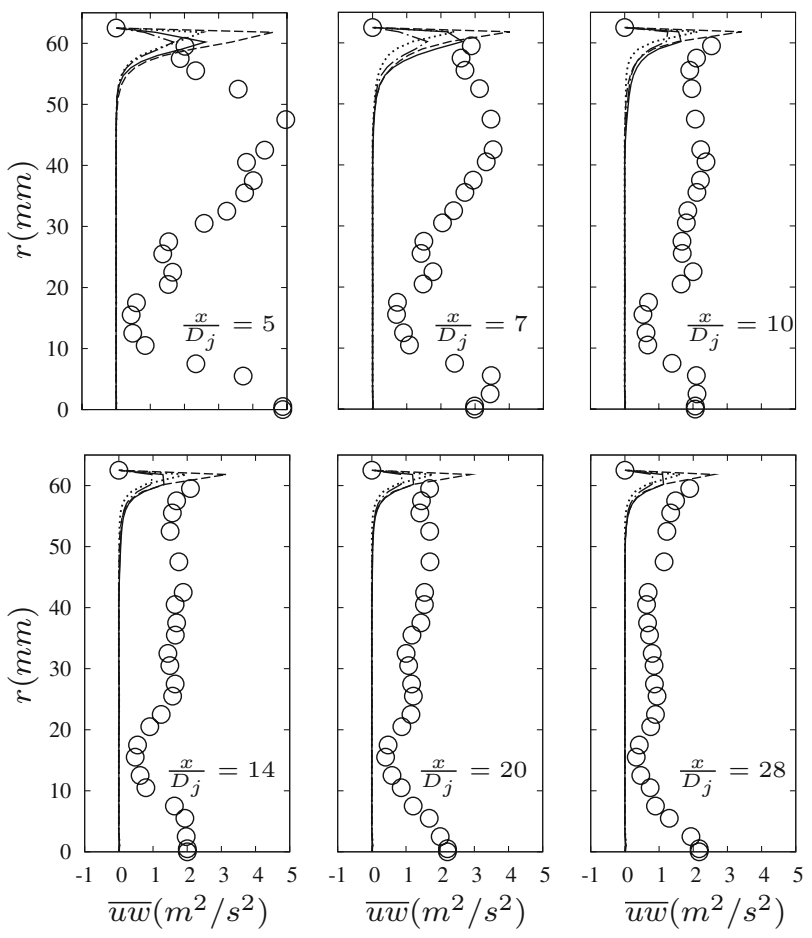

Fig. 19 Predicted and measured cross-stream profile of shear-stress component $\overline{u w}$ obtained with different pressure-strain models. Symbols are as in Fig. 2
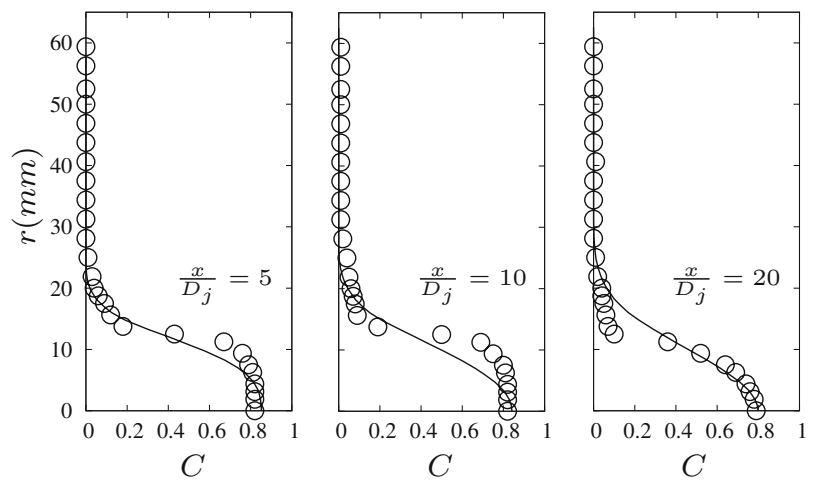

Fig. 20 Predicted and measured cross-stream profile of concentration
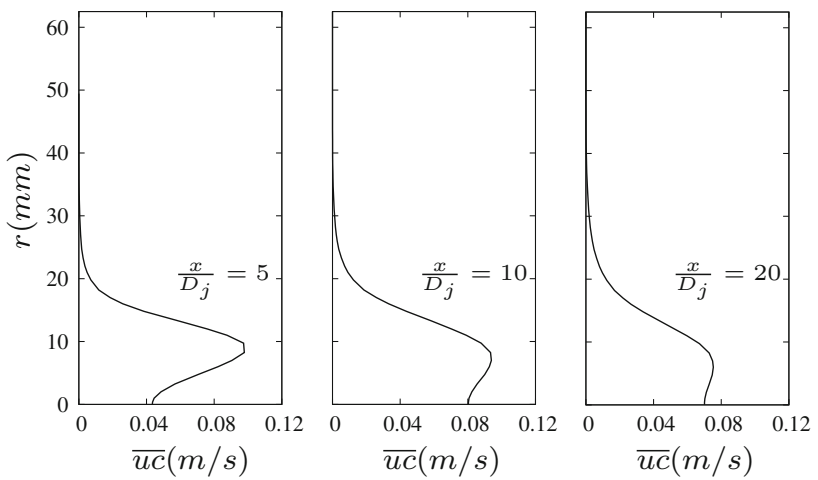

Fig. 21 Predicted cross-stream profile of scalar flux $\overline{u c}$ 


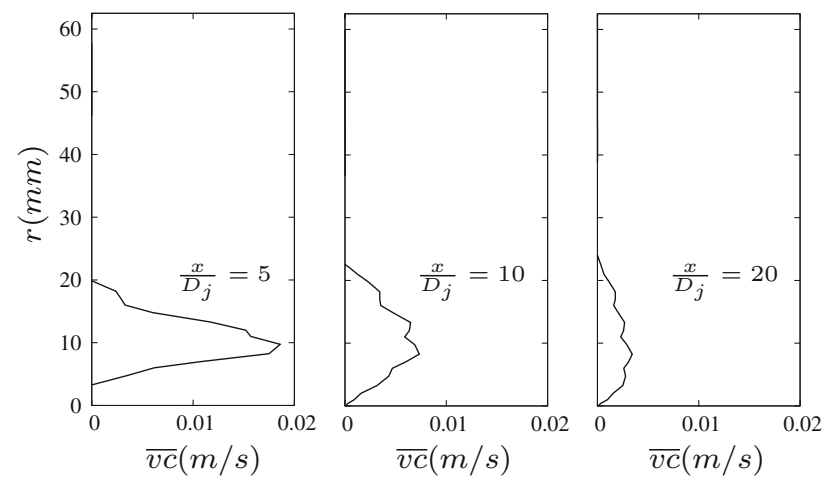

Fig. 22 Predicted cross-stream profile of scalar flux $\overline{v c}$

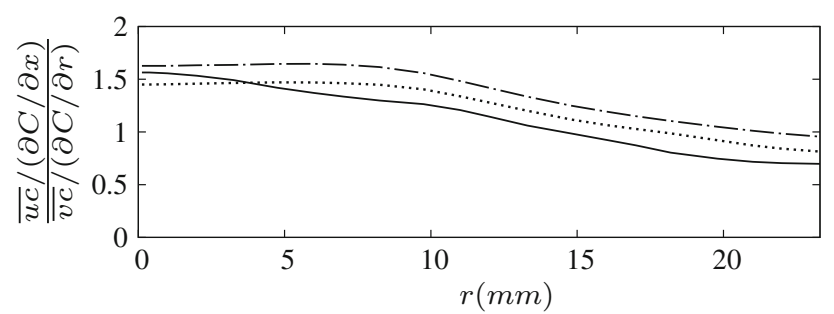

Fig. 23 Predicted anisotropy of turbulent diffusivities. - $: x / D_{j}=5 ; \cdots \cdots: x / D_{j}=10 ;-\cdots \cdots \cdot$-..-: $x / D_{j}=20$

\section{Conclusions}

Experimental data from weakly- and strongly-swirled turbulent jets were used to assess the performance of a model for the pressure-strain correlations which satisfies the principle of Material Frame Indifference. The model is simpler than others of its category and, moreover, has been calibrated so as not to require the use of wall-damping functions. In order to put the model's performance in proper perspective, comparisons were also made with three other pressure-strain models. The new model was found to produce overall fairly good results for both weakly- and strongly-swirled cases. In the latter, which involved confined co-axial jets with swirl imparted to the outer stream, the new model's ability to capture the effects of a solid wall on the turbulence intensity close to it without the use of wall damping was clearly demonstrated. Predictions were also obtained for the case of a non-swirling helium jet discharged into an outer, swirling, air stream. The turbulent mass fluxes were computed by using a differential flux transport model used in conjunction with the objective model for pressure-strain correlations. Good predictions were obtained for the profiles of mean concentration. Analysis of the predicted scalar fluxes showed turbulent diffusivities that were strongly anisotropic.

Acknowledgments Anja Vogler gratefully acknowledges the financial support provided by the MTU Studien-Stiftung and the Erich-Becker-Stiftung.
Open Access This article is distributed under the terms of the Creative Commons Attribution Noncommercial License which permits any noncommercial use, distribution, and reproduction in any medium, provided the original author(s) and source are credited.

\section{Appendix: Grid convergence index}

The steps involved in obtaining the Grid Converge Index used above to quantify the numerical uncertainties in the predictions are as follows:

1. Generate three different grids and calculate the grid refinement factors:

$r_{21}=\left(\frac{N_{1}}{N_{2}}\right)^{\frac{1}{3}} \quad r_{32}=\left(\frac{N_{2}}{N_{3}}\right)^{\frac{1}{3}}$

where $N$ refers to the number of cells in a fine grid $\left(N_{1}\right)$, medium grid $\left(N_{2}\right)$ or coarse grid $\left(N_{3}\right)$ and $r$ refers to the grid-refinement factors for the fine-medium grids $r_{21}$ and the medium-coarse grids $r_{32}$.

2. Calculate the differences in one or more key flow variables resulting from the use of the different grids:

$e_{21}=\phi_{2}-\phi_{1} \quad e_{32}=\phi_{3}-\phi_{2}$

In the present study the key variables $\phi$ were chosen to be the cross-stream profiles of mean axial velocity, and the ratio of the axial velocity at the centerline $U_{c}$ and the centerline velocity at the inlet plane $U_{0}$ in a fine grid $\left(\phi_{1}\right)$, medium grid $\left(\phi_{2}\right)$ or coarse grid $\left(\phi_{3}\right)$.

3. Calculate the apparent order $p$ using fixed-point iteration:

$p=\frac{1}{\ln r_{21}}|\ln | \frac{e_{32}}{e_{21}}|+q(p)|$

$q(p)=\ln \left(\frac{r_{21}^{p}-s}{r_{32}^{p}-s}\right)$

$s=\operatorname{sign}\left(\frac{e_{32}}{e_{21}}\right)$

4. Calculate the approximate relative error:

$e_{a}^{21}=\left|\frac{\phi_{1}-\phi_{2}}{\phi_{1}}\right|$

5. Calculate the fine grid converge index:

$G C I^{21}=\frac{1.25 e_{a}^{21}}{r_{21}^{p}-1}$

\section{References}

1. Wicker RB, Eaton JK (2001) Structure of a swirling, recirculating coaxial free jet and its effect on particle motion. Int J Multiphase Flow 27:949-970 
2. Tang G, Yang Z, McGuirk JG (2002) Large Eddy Simulation of isothermal confined swirling flow with recirculation. Eng Turbul Model Exp 5:885-894

3. Freitag M, Klein M (2005) Direct Numerical Simulation of a recirculating swirling flow. Flow Turbul Combust 75:51-66

4. Freitag M, Klein M, Gregor M, Geyer D, Schneider C, Dreizler A, Janicka J (2006) Mixing analysis of a swirling recirculating flow using DNS and experimental data. Int $\mathbf{J}$ Heat Fluid Flow 27:636-643

5. Launder BE, Morse AP (1979) Numerical prediction of axisymmetric free shear flows with a Reynolds stress closure. In: Durst $\mathrm{F}$ et al (eds) Turbulent shear flows I. Springer-Verlag, Berlin, pp 279-294

6. Gibson MM, Younis BA (1986) Calculation of a swirling jet with a Reynolds stress closure. Phys Fluids 29:38-48

7. Speziale GC (1998) A review of material frame indifference in mechanics. Appl Mech Rev 51:489-504

8. Dafalias Y, Younis BA (2009) An objective model for the fluctuating pressure-strain-rate correlations. ASCE J Eng Mech 135(9)

9. Smith GF (1971) On isotropic functions of symmetric tensors, skew-symmetric tensors and vectors. Int J Eng Sci 9:899-916

10. Speziale CG, Sarkar S, Gatski T (1991) Modeling the pressurestrain correlation of turbulence: an invariant dynamical systems approach. J Fluid Mech 227:245-272
11. Dafalias Y, Younis BA (2007) Objective tensorial representation of the pressure-strain correlations of turbulence. Mech Res Commun 34:319-327

12. Launder BE, Reece GJ, Rodi W (1975) Progress in the development of a Reynolds stress turbulence closure. J Fluid Mech 68:537-566

13. Gibson MM, Launder BE (1978) Ground effects on pressure fluctuations in the atmospheric boundary layer. J Fluid Mech 86:491-511

14. Malin MR, Younis BA (1990) Calculation of turbulent buoyant plumes with a Reynolds stress and heat flux transport closure. Int J Heat Mass Transf 33:2247-2264

15. Leonard BP (1979) A stable and accurate convective modeling procedure based on quadratic upstream interpolation. Comput Methods Appl Mech Eng 19:59-98

16. Roache PJ (1994) Perspective: a method for uniform reporting of grid refinement studies. J Fluids Eng 116:405-413

17. So RM, Ahmed SA, Mongia HC (1984) An experimental investigation of gas jets in confined swirling air flow. NASA CR-3832

18. Ahmed SA, So RM (1986) Concentration distributions in a model combustor. Exp Fluids 4:107-113

19. Younis BA, Speziale CG, Clark TT (2005) A rational model for the turbulent scalar fluxes. Proc R Soc 461:575-594

20. Wei X, Zhang J, Zhou L (2004) A new algebraic mass flux model for simulating turbulent mixing in swirling flow. Numer Heat Transf B 45:283-300 